\title{
The Analysis on the Application of SCT in Academic English Teaching
}

\author{
Yuehong Wei ${ }^{1, a,{ }^{*}}$, Chungang Miao ${ }^{2, b}$ \\ ${ }^{1}$ English Department, North China Electric Power University, Baoding, Hebei, China \\ ${ }^{2}$ Department of political and law, North China Electric Power University, Baoding, Hebei, China \\ aiowalily@163.com, ’mcg602@gmail.com
}

${ }^{*}$ Corresponding author

\begin{abstract}
Since 1890s, SCT has been the new study topic in Second Language Acquisition. SCT gives the deep insight into the social elements and historical and cultural elements in the process of language learning and improving the cognitive ability. Meanwhile, with the tendency of internalization, intercultural communicative ability becomes more and more important especially in the academic communication. This paper will work on the application of SCT in the academic English teaching, focusing on the study of teaching methods in order to help student improve their learning ability in academic fields.
\end{abstract}

Keywords: SCT, academic learning, internalization, mediation, activity theory

\section{社会文化理论在学术英语教学中的运用探讨}

\author{
魏月红 ${ }^{1, a,{ }^{*}}$, 苗春刚 ${ }^{2, b}$, \\ 1 英语系, 华北电力大学, 保定, 河北, 中国 \\ 2 法政系, 华北电力大学, 保定, 河北, 中国

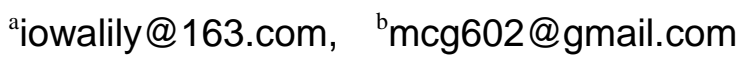

*通讯作者

中文摘要：自上世纪八十年代以来，社会文 化理论已经成为二语习得 (Second Language Acquisition）研究的新兴领域。社会文化理论 注重语言学习过程中社会因素和历史文化对 学习者语言认知能力的影响, 强调语言的调 控中介作用和学习者的内化过程。本文将以 社会文化理论的核心概念为理论指导, 探讨 我国学术英语教学的有效方法, 其目的是让
学生达到学有所用、学以致用及以用促学的 实效英语学习状态。

关键词: 社会文化理论, 学术英语教学, 内 化, 中介, 活动理论 


\section{1. 引言}

自上世纪八十年代以来, 社会文化理论已经 成为二语习得 (Second Language Acquisition) 研究的新兴领域。社会文化理论由俄国心理 学家维果斯基提出, 并由最具代表性的美国 宾夕法尼亚州立大学的詹姆斯兰多夫教授引 入第二语言习得领域, 成为第二语言习得领 域研究的一个新的视角。社会文化理论注重 语言学习过程中社会因素和历史文化对学习 者语言认知能力的影响, 强调语言的调控中 介作用和学习者的内化过程。社会文化理论 能够解决我国目前英语教学中存在的问题, 比如英语学习只限于课本知识, 缺少实际语 言交际场景和练习; 英语学习只限于通用英 语语境, 不能解决国际学术交流问题等。当 前我国学术英语教学刚刚起步, 但是其实效 性却引起教育学者的担忧。在当今经济全球 一体化发展态势下，国际交流日益频繁，教 育高素质人才的基本要求即是国际沟通交流 能力, 这里的沟通交流能力已经不仅仅局限 于日常的沟通, 而是更为广阔的专业学术交 流和专业学术学习能力, 所以语言能力则在 这样的社会发展态势下显得尤为重要, 这也 要求我国英语教学应该以社会实际交往能力 为导向。本文将以社会文化理论的核心概念 为理论指导, 探讨我国学术英语教学的有效 方法, 其目的是让学生达到学有所用、学以 致用及以用促学的实效英语学习状态。

\section{2. 社会文化理论}

\section{1 中介理论 (Mediation)}

中介理论是维果斯基社会文化理论的核 心概念。维果斯基 (1978) 认为高级认知功 能的发展依赖于人与社会环境的互动, 以及 语言与其他社会文化符合产物的调节关系, 也就是人的认知活动是通过文化制品或符号 进行中介, 进而达到发展提高的过程, 这也 就是中介理论的核心内容。根据 Lantolf he Thorne(2006)的观点, L2 学习的中介理论是 指: 1) 在社会互动中与他人的中介 (如人与 人之间的中介或称为他人中介; interpersonal mediation or other regulation）；2）通过社会 文化产物的中介或称作事物中介（mediation by artifacts or object regulation, 如通过网络);
3) 通过自我语言（private speech, 或称自我 话语）的自我中介（mediation by self or self regulation)（转引自秦丽莉, 戴炜栋, 2013)。 在这里, 语言符号是最基本的调节工具, 通 过语言的调节，语言学习者的认知机能达到 从低级到高级的转化。

\section{2 内化理论}

维果斯基提出这一理论目的是克服二元 理论, 其方法为融合生态和社会因素为一个 统一的理论框架。内化理论就是建立一个能 够控制人类天赋的一种机制，人类通过社会 文化体验的中介形成新的思维活动, 以此来 控制人类自然的心理功能, 这一过程即是内 化过程。内化过程协商发展过程, 它包括对 人际关系的再构建过程, 在这个过程中, 人 和环境的关系也再次构建, 并且能够促进将 来新的人与环境的重新构建。

\section{3 活动理论（Activity Theory）}

维果斯基（1978：32）提出 “人的心理 过程的变化与他的实践活动过程的变化的是 一样的”。活动理论是当代研究领域一种有效 的研究方法, 为人类活动提供了认知导向, 从生态角度和文化活动角度提供了认知导 向。活动理论认为人的高级认知功能是在社 会活动实践中发展起来的, 研究重点是个体 或者群体在特殊情境下采取哪种活动, 而不 只是关注语言的技能、信息的处理和概念的 应用( Vygotsky，1978)。活动理论为人类的 行为和活动提供了认知学的解释, 同时也提 供了一个全新解释人类发展的理论分析视 角。因此活动理论把人的行动视为社会和个 体相互影响的动态系统, 认为活动是社会多 层面合作的过程, 不能以教师和学生任何一 方为中心, 只能是彼此的共同合作( Lantolf \& Thorne, 2006)。活动理论构架了一座从 单个对象到社会活动的桥梁, 进一步强调了 社会、历史及文化在语言学习中的作用。

\section{4 最近发展区和脚手架/支架（Scaffold）}

Vygotsky 的最近发展区( ZPD) 是指学 习者个体独立发展的语言水平与在高水平的 合作者帮助下发展的语言水平之间的距离 ( Lantolf \& Thorne，2006)。最近发展区符 合人类认知的过程, 能够对实际教学产生有 
效的指导作用。但是, 最近发展区的学习内 容比较难以把控, 这是该理论应用中的难点 所在。支架理论是指任何成人儿童或者专家 新手的相互协作的行为 (Lantolf \& Thorne, 2007)。Wertsch (2007)进一步指出, 支架可以 是同伴之间的互动和一个集体之间的互动。 该理论和最近发展区综合运用能够对学习者 起到有效的推动作用, 符合人类认知能力有 低级到高级的演变规律。

\section{3. 学术英语教学}

学术英语教学应时代的需要而在中国英 语教学中越来越受到重视。但是在传统英语 教学的影响下, 其发展进程确遇到很大的阻 力。教师的教学理念要改变, 学生的学习方 法要改变, 而英语学习是一个长线任务, 有 前后承接关系, 比如大学生在学英语时总会 受到中学时学习习惯的影响。要解决这个问 题首先要区分 “通用英语” 和 “学术英语” 的区别, Scarcella（2003：9）把学术英语定 义为 “在专业书籍中使用的英语, 具有与学 科有关的语言特点”。两者互有不同, 但又彼 此联系。“学术英语” 是 “通用英语” 的高级 语言形式, “通用英语” 是 “学术英语” 的语 言基础。学术英语教学应该以语言实际应用 能力为目标, 使学习者能够学以致用, 使学 习者感到学有所用, 并且能够以用促学。社 会文化理论恰恰能够知道学术英语教学达到 这一教学目标, 因为社会文化理论主张社会 交流, 重视文化历史等动态因素对学习者认 知能力的影响。

本文主要从学术英语阅读和写作两个方 面, 论证社会文化理论的实际应用。其中包 括: 教学内容, 教学活动, 评价方法, 教学 反思等环节。

\section{4. 核心概念的实际应用}

\section{1 中介和内化理论的应用}

在这一阶段, 重要培养学生的“学术英语” 学习认同感。主要通过教师、自我语言中介 建立对学术英语学习的正确认识, 防止在学 习过程中出现学习目标不明确, 学习动力不 足等问题。首先, 教师介绍学术英语的概念, 重要性, 学习方法, 学习的主要方面; 其次,
通过提问的方式, 了解学生对学术英语的理 解, 会在学术英语学习中遇到哪些问题等; 然后, 选择学生感兴趣的学术英语文章、学 术问题及学术会议视频等材料进行学习分 析, 这一环节尤为重要, 注意学习材料的难 易程度和题材必须适合学习者, 否则将会打 消学生的学习积极性和主动性; 再然后, 则 是到了 “师傅领进门, 修行在个人” 的环节, 在经过前一阶段的教师调节和个人调节后, 开始进入内化阶段, 同样要选取适合的学习 材料, 但这一阶段的学习材料基本由学生自 己来选择, 教师可以提供帮助和辅导, 学生 在这一过程中会出现一些问题, 教师要积极 的引导, 使其能够自我调节, 对所学知识由 外部知识转为内部知识, 只有通过学习者自 身的体验和反复思考, 才能够达到内化的目 的。最后, 评价环节采用教师评价和同伴互 评, 因为每个学习者都有认真的学习体验, 所以他们心中对于学习的效果都有很清楚的 认识, 也就能够保证评价的公正性。

\section{2 活动理论的实际应用}

活动理论强调语言学习的交互性, 即在 实际社会交际中学习语言。这对于学术英语 学习尤为重要, 因为学术英语学习的目的就 是学以致用, 那么脱离实践的学习则无法达 到这一目的。这里的活动则指的是个人与社 会的一种交互过程, 在学术英语学习中, 我 们不能脱离实际社会实践来学习, 所以在这 一环节中, 教学内容强调真实情景的设立, 比如真实论文议题写作、学术会议实际演练, 鼓励学生大胆尝试, 敢于面对挑战和问题, 通过实际的操作练习, 提高自己的语言认知 能力, 并且在这一过程中也会涉及中介和内 化的参与。每个实际操练环节后, 都会有实 际学术英语成绩反馈, 首先就是真实参与的 结果, 再次是教师就学生自身水平给出的评 价, 比如是否相对以前有所进步、哪一方面 是薄弱环节等, 同伴互评, 同伴可以根据自 己的体验对对方做出客观的评价, 还可以取 长补短, 这样更能够激发学习的认同感和同 理心。

\section{3 最近发展区域和脚手架的实际应用}

最近发展区域和脚手架理论为教师指导 学生提供了很好的依据和途径。首先, 学习 
材料的选取要适度, 在这里指的是要接近学 生水平, 但要高于学生水平, 也就是让学生 蹦一蹦能够达到的水平, 比如, 学术会议需 要接近学生的专业领域, 但是要和学生所学 内容相关, 能够通过思考和查找资料完成某 一学术问题的研究, 这样可以达到开阔学生 思维和开阔视野, 提高研究能力的目的。那 么, 教师如何是学生完成 “蹦一蹦” 的任务 呢? 那就是需要为学生搭建脚手架, 帮助学 生建立一个通向目标的基本知识构架, 当然, 只限于一个构架, 可能需要学生加 “砖” 加 “瓦” 来建造一条路, 在这个过程中学生的 水平自然得到提高, 也就自然的达到了设定 的学习目标。

\section{5. 结束语}

学术英语学习对于中国学生来说是非常 必要的, 但是由于学生的个体差异存在很大 的不同, 所以要求教师要不断地研究教学方 法, 对学生给予正确的引导, 包括学习方法 的引导, 学术发展的引导, 和语言认知能力 提高途径的引导。本文尝试从社会文化理论 角度, 对学生英语教学提出了以上几点教学 尝试, 强调了实际演练的重要性, 充分考虑 教学环节中的教学材料选取和教师中介引导 的作用, 这些应该是学术英语教学中需要考 虑和仔细研究的教学环节。由于该论文只限 于理论探讨, 缺少实证研究的为佐证, 期待 相关实证研究能够尽快完成, 以便更好地服 务于学术英语教学改革的顺利进行。

\section{致谢}

This research was supported by Social Sciences Fund of Hebei Province (Grant \# HB13YY008) and the Educational Department Fund of Hebei Province (Grant\# SZ141147) , by "the Fundamental Research Funds for the Central Universities (Grant \# 2014MS179)" and funded by Teaching reform project in Hebei Province.

\section{References}

[1]. Vygotsky, L. S. 1978. Mind in Society : The Developmentof Higher Psychological Process. Cambridge, MA : Harvard
University Press.

[2]. Lantolf, J. P. Language emergence: implications for Applied Linguistics a sociocultural perspective [ J ] 2006. Applied Linguistics, 27( 4) : 717 - 728 .

[3]. Han Dawei, Deng Qi. 2013. Dynamic or interactive? - - the application of Dynamic system theory and SCT in SLA [J]. CAFLE, 2013(5):10-15.

[4]. Lantolf, J. \& S. Thorne. 2006. The Sociogenesis of Second Language Development [ $\mathrm{M}]$. Oxford: Oxford University Press. 